\title{
Heat Transfer Correlations for a Composite PCM Based 72 Pin Fin Heat Sink with Discrete Heating at the Base
}

\author{
R. Srikanth ${ }^{1}$ - C. Balaji ${ }^{1}$
}

Received: 7 February 2017/ Accepted: 16 June 2017/Published online: 11 July 2017

(C) Indian National Academy of Engineering 2017

\begin{abstract}
This paper reports the results of an experimental investigation on the thermal performance of a phase change material based cuboidal heat sinks with 72 pin fins on the inside to act as thermal conductivity enhancers. The heat sink base is heated with four equal sized heaters. The total power of the heaters is fixed, while the individual wattage can vary. First, in house experiments are conducted for different combinations of power. Based on the experimental results, correlations are then developed for the characteristic time for phase change with natural convection as a function of the individual heat input values. Additional experiments are conducted to validate the developed correlation and a good agreement is found. The correlations developed are useful for future optimization studies.
\end{abstract}

Keywords Phase change material $\cdot$ Heat sinks $\cdot$ Electronic cooling $\cdot$ Melting $\cdot$ Convection

\section{List of symbols}

$\begin{array}{ll}\text { Abbreviations } \\ A l & \text { Aluminum } \\ A m b & \text { Ambient } \\ \text { Exp } & \text { Experimental }\end{array}$

C. Balaji

balaji@iitm.ac.in

1 Heat Transfer and Thermal Power Laboratory, Department of Mechanical Engineering, Indian Institute of Technology Madras, Chennai 600036, India
PCM Phase change material

$R^{2} \quad$ Correlation coefficient

TCE Thermal conductivity enhancers

\section{Notations}

Fo Fourier number

$g \quad$ Acceleration due to gravity, $9.81 \mathrm{~m} / \mathrm{s}^{2}$

$H \quad$ Height of the heat sink, m

$k \quad$ Thermal conductivity, $\mathrm{W} / \mathrm{mK}$

$k_{s} \quad$ Thermal conductivity of Aluminum, W/mK

$L \quad$ Latent heat of PCM, $\mathrm{kJ} / \mathrm{kg}$

$\mathrm{Nu} \quad$ Nusselt number

$q_{b} \quad$ Heat flux at the base, $\mathrm{W} / \mathrm{m}^{2}$

$P, Q$ Power input to the heater

$R a \quad$ Rayleigh number

Ste Stefan number

$t_{c} \quad$ Cooling time, $\mathrm{s}$

$T_{m} \quad$ Melting point, $\mathrm{K}$

$\mathrm{T}$ Temperature, $\mathrm{K}$

$t_{s} \quad$ Time to reach set point temperature, $\mathrm{s}$

$t_{s e} \quad$ Sensible heating time(pre melting), $\mathrm{s}$

$t_{L} \quad$ Latent heating time, $\mathrm{s}$

$t \quad$ Thickness of the heat sink, $\mathrm{m}$

W Half length of the heater, $m$

\section{Greek symbols}

$\alpha \quad$ Thermal diffusivity, $\mathrm{m}^{2} / \mathrm{s}$

$\beta$ Thermal expansion coefficient of PCM, $\mathrm{K}^{-1}$

$\gamma \quad$ Liquid fraction

$v \quad$ Kinematic viscosity, $\mathrm{m}^{2} / \mathrm{s}$

$\rho$ Density of PCM, $\mathrm{kg} / \mathrm{m}^{3}$

$\rho_{s} \quad$ Density of aluminum, $\mathrm{kg} / \mathrm{m}^{3}$

$\tau$ Characteristic time for phase change with convection, (Fo.Ste) $/ R a^{0.25}$ 


\section{Introduction}

Rapid miniaturization of electronic devices leads to very high power dissipation rates, which in turn result in ever increasing device temperatures. The reliability of an electronic device is a strong function of temperature, in view of which its temperature control is highly critical. Conventional cooling techniques like air cooling and water cooling are not suitable when compactness of the electronic devices becomes an important aspect of the design. The main objective in the thermal design of any electronic equipment is to keep the device operating temperature within safe limits to ensure reliability. Phase change material (PCM) based cooling technique is a passive type cooling that offers several advantages over conventional cooling techniques, particularly in intermittent duty cycles due to the large latent heat of the PCM. The desirable properties of such a PCM are

1. high latent heat of fusion

2. high specific heat capacity

3. good thermal conductivity

4. suitable melting temperature (less than the set point temperature)

5. negligible volume change during phase change

6. chemically stability.

For example, ice as a latent heat storage material has a very high enthalpy of $334 \mathrm{~kJ} / \mathrm{kg}$, but due to its high volume expansion, is not suitable for storage in a cavity like heat sink.

The major drawback of a PCM based heat sink is, however, its very low thermal conductivity. The thermal conductivity of a typical PCM like $n$-eicosane is $0.23 \mathrm{~W} /$ $\mathrm{mK}$ in the solid state and is much lower in the liquid state. In a PCM based heat sink, the PCM layer located far away from the solid surface can utilize the latent heat only after the adjoining layer of PCM is fully molten. The low thermal conductivity of PCM, results in high thermal resistance to the flow of heat, which in turn results in a high temperature gradient within the PCM heat sink in the direction of flow of heat. Addition of thermal conductivity enhancers (TCE) can help overcome the high resistance offered by the PCM to the flow of heat. The addition of such TCEs ensures lower temperature gradient and effective utilization of the latent heat. In general, the desirable output in a PCM based heat sink would be a high latent heat time during the charging cycle. Latent heat absorption phenomenon associated with the melting PCM can be used to delay the temperature rise of the heat sink subjected to high heat flux, to a reasonable extent, and hence is a key player in the accomplishment of our goal of maximizing the time to reach a set point temperature $\left(42{ }^{\circ} \mathrm{C}\right)$.
Pillai and Brinkworh (1976) considered the use of phase change material in energy storage systems and summarized the critical points in the selection of PCMs for particular application. The authors also emphasized in their study on the optimization importance of during the design of PCM based energy storage systems. The authors concluded that PCM offers a convenient means of storing low grade thermal energy within compact systems Sasaguchi and Viskanta (1987) experimentally investigated the simultaneous melting and solidification of PCM around two horizontal cylinders. The authors concluded that the spacing between the cylinders has a significant impact on the shape of the melt front. Ho and Viskanta (1984) reported basic heat transfer data during melting from an isothermal wall. The PCM used in the study was $n$-octadecane and the enclosure shape was rectangular. During the experiments shadowgraph technique was used to capture the position of the solid liquid interface position at various time instants and a corresponding numerical model was also developed and good agreement between the two was found. The effect of Rayleigh number on the heat transfer coefficient during the melting was also reported. Webb and Viskanta (1985) attempted to determine the characteristic length scale for calculating the heat transfer parameters during the melting process. They concluded that the height of the enclosure is not an appropriate length scale as the molten PCM shape keeps changing with time. Jany and Bejan (1988) determined the appropriate length scales for melting heat transfer with natural convection inside an enclosure.

Akhilesh et al. (2005) proposed a thermal design procedure for the composite heat sinks (CHS) for effective utilization of thermal storage energy which helps in stretching the operation time of the heat sink.

Nayak et al. (2006) investigated the effectiveness of the TCEs to improve the overall thermal conductivity of the PCM by developing a numerical model. The control volume approach was used to solve the governing equations.

Baby and Balaji (2012) conducted experimental investigations on PCM based finned heat sink. The PCM used in the study was $n$-eicosane. The effect of plate fin and pin fin on the thermal performance of the heat sink was studied.

Baby and Balaji (2013) performed optimization studies based on neural network coupled with Genetic algorithm based optimization on finned heat sinks using $n$-eicosane as the PCM. The neural network was driven purely by the experimental data and an optimum configuration of the heat sink was arrived at.

Fan et al. (2013), experimentally, investigated the effect of internal fins on the performance of the PCM based heat sinks. The thermal performance was compared with an unfinned heat sink to determine the enhancement in heat transfer due to the addition of fins. 
Shokouhmand and Kamkari (2013), experimentally visualized the temperature field and melt front shape and position during the solid liquid phase change of lauric acid. The experiments were conducted in a rectangular heat sink. They concluded that during the end of the convection dominated melting process, the bulk temperature of PCM increased and high temperatures are found at the upper part of enclosure.

Srikanth and Balaji (2017) experimentally investigated the effect of discrete heat loading in a 72 pin fin heat sink with discrete heat load at base. Preliminary experiments were conducted and $n$-eicosane was used as the PCM. The authors concluded that a single plate heater and discrete heater with uniform heat distribution show the same performance. Srikanth et al. (2015) performed a multi objective geometric optimization on a pin fin matrix heat sink using Non-dominated sorting genetic algorithm. The authors developed a matched up numerical model and constructed an artificial neural network whose output was the fitness function for the evolutionary algorithm. The obtained Pareto optimal solutions obtained were validated with the developed numerical model developed and the agreement between ANN output and the numerical model output was found to be good.

From the review of literature presented above, it is evident that the use of phase change material (PCM) based composite heat sinks for electronic cooling is a well established field of research. Several studies have focused on the effect of geometry, orientation on the performance of the heat sink. However, in applications, a single uniform heat generating element is often not the case. Studies on the discrete heat loading on a PCM based heat sink with TCEs are scarce in literature. In view of this, the objective of this work is to conduct experiments to investigate the effect of discrete heat loading on the melting of PCM enclosed in a rectangular heat sink with 72 internal pin fins. The total wattage remains 6 and $4 \mathrm{~W}$ throughout the study. The set point temperature is taken as $315.2 \mathrm{~K}$.

\section{Experimental Setup}

\section{Heat Sink Design}

The heat sink used in the present study is made of aluminium. A picture of the heat sink is shown in Fig. 1. The 72 pin fins are fabricated using the electro discharge machining process. The heat sink cavity has a depth of $20 \mathrm{~mm}$. The fins have a cross section of $2 \mathrm{~mm} \times 2 \mathrm{~mm}$. The overall dimensions and the spacing between the fins are same as in (Srikanth and Balaji 2017). The heat sink has a wall thickness of $7 \mathrm{~mm}$. All the four side of the walls and the bottom face of the heat sink are covered with cork to minimize the heat loss to the

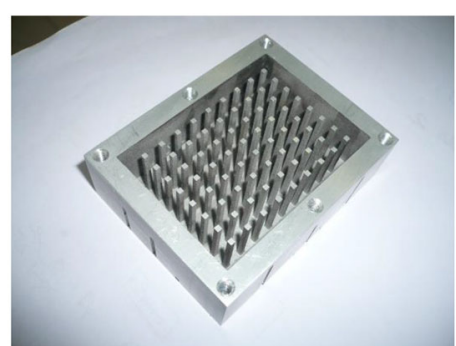

(a)

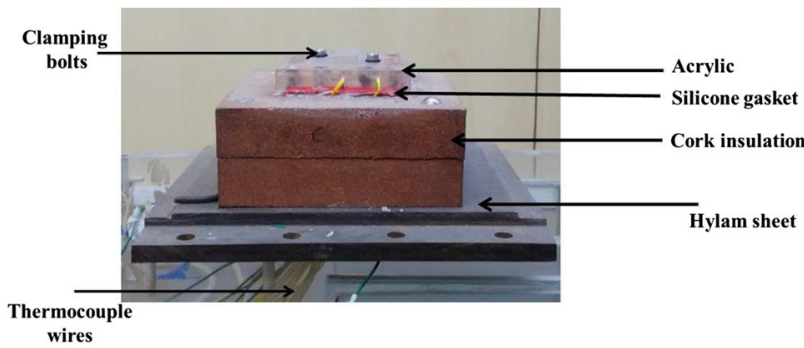

(b)

Fig. 1 Picture showing the heat sink with pin fins a before assembly b after assembly

ambient. A $2 \mathrm{~mm}$ slot is given at the bottom face of the heat sink to accommodate the heaters. The top face of the heat sink is covered with acrylic by means of bolts. A silicon gasket of $2 \mathrm{~mm}$ thickness is placed between the acrylic and the top face of the heat sink to avoid any spillage or leak of the PCM. The torque on the bolts is very critical to avoid any PCM leak to the outside.

\section{Heater Design}

To mimic the heat dissipating units in portable electronic devices four discrete heaters were placed at the bottom of the heat sink. The positions of the heaters are shown in

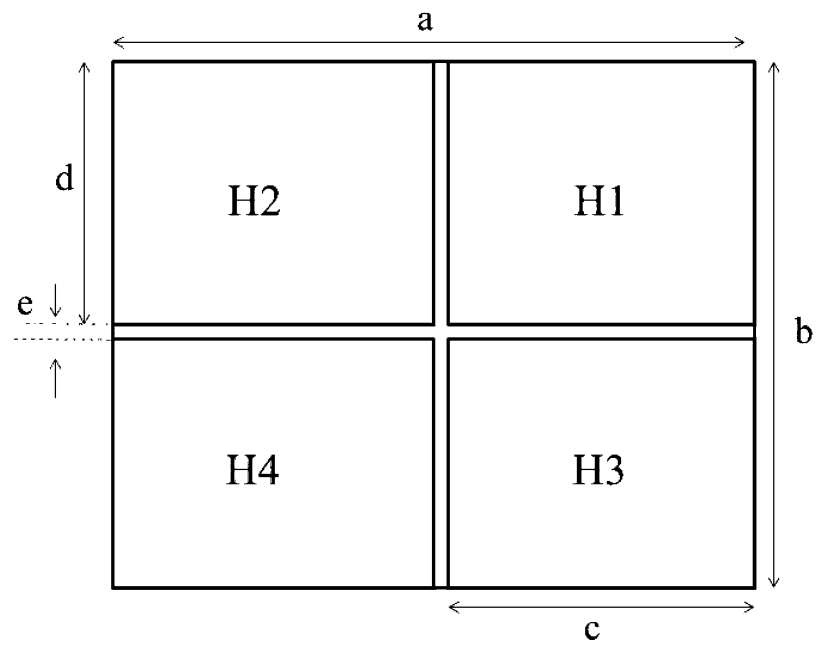

Fig. 2 Picture showing the dimensions of a discrete heater 
Table 1 Heater dimensions for Fig. 2

\begin{tabular}{lll}
\hline SI. no & Dimension & Value in $\mathrm{mm}$ \\
\hline 1 & $\mathrm{a}$ & 60 \\
2 & $\mathrm{~b}$ & 42 \\
3 & $\mathrm{c}$ & 29 \\
4 & $\mathrm{~d}$ & 20 \\
5 & $\mathrm{e}$ & 2 \\
\hline
\end{tabular}

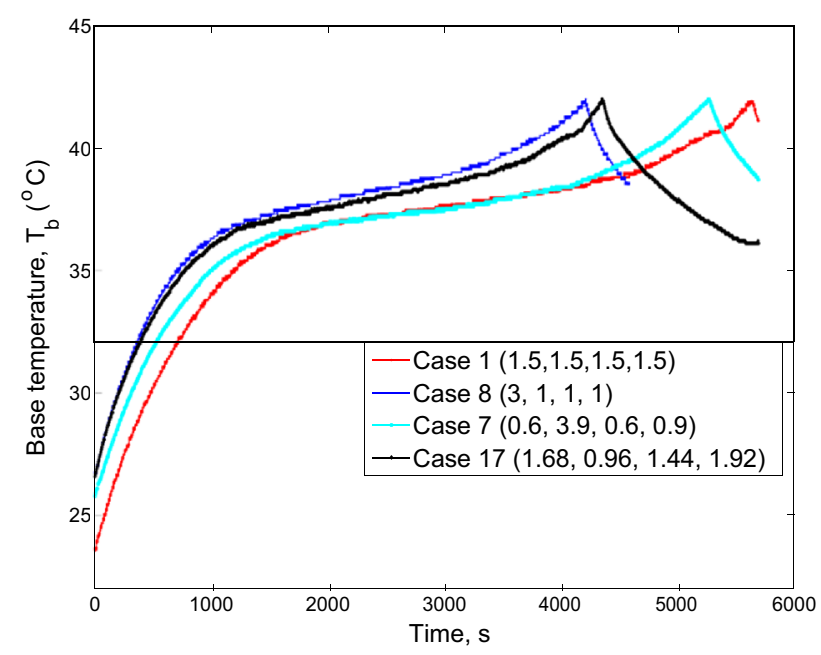

Fig. 3 Variation of wall temperature with time

Fig. 2. The heaters are made of mica sheet over which the nichrome wire is wound. The size of the mica sheet is $29 \mathrm{~mm} \times 20 \mathrm{~mm}$.

The heater area available at the base is $60 \times 42 \mathrm{~mm}^{2}$. This area is subdivided into four equal areas into which the discrete heater were placed. The dimensions of the heater are as same as in (Srikanth and Balaji 2017) and are shown in Fig. 2 and Table 1. A gap of $1 \mathrm{~mm}$ is maintained between the heaters to avoid any electrical contact. The diameter of the nichrome wire is $0.2 \mathrm{~mm}$. Nichrome is chosen as it is highly corrosion resistant and does not oxidize at higher temperatures. The nichrome wire is coiled in a zig zag manner over the mica sheet. The output of the heater design process is the length of the heater. The resistivity of the nichrome wire used in the present study is $1.5 \times 10^{-6} \Omega \mathrm{m}$. The heat generated by the heating element depends on the current passing through the wire which again depends on resistance, length, and cross section of the wire. The DC power source used in the present study has a voltage rating of $0-10 \mathrm{~V}$ and current rating of $0-1.25 \mathrm{~A}$.

$P=\frac{V^{2}}{R}$.

To obtain a minimum power of $0.5 \mathrm{~W}$ and a maximum power of $2 \mathrm{~W}$, the length of the nichrome wire for each discrete heater was determined to be $440 \mathrm{~mm}$.

\section{Thermocouple Positions}

For the present research, a total of 16 calibrated k-type thermocouples are used for measurement of temperature at various locations inside the heat sink. Calibration is carried out using a constant temperature water bath in the temperature range of $30-70{ }^{\circ} \mathrm{C}$, in both heating and cooling cycle. Six thermocouples $\left(T_{1}\right.$ and $\left.T_{2}\right)$ are placed at the corresponding milled slots on the heat sink base. One thermocouple was placed at the centre slot of each discrete heater. Two thermocouples are placed at the centre slot of the base of the heat sink. Four thermocouples, each of which are placed on each wall. To measure the inside PCM temperature, two thermocouples, each were placed at 5,10 , $15 \mathrm{~mm}$ from the base inside the PCM. One thermocouple is placed in still air to measure the ambient temperature. All sixteen thermocouples are bonded to the heat sink using Omega Araldite epoxy.

\section{Uncertainty Analysis}

The uncertainty associated with the measurement of fundamental quantities like the voltage and current can be taken as the least counts of the corresponding measurement devices. In so far as the temperature measurement is concerned, based on the calibration, it can be inferred that value of temperature is accurate within $\pm 0.2{ }^{\circ} \mathrm{C}$. However, the derived quantity power, which is a product of the voltage and current, requires an uncertainty calculation, the results of which are shown in Table 2.

The uncertainty in power can be calculated as

$\sigma_{p}= \pm \sqrt{\left(\frac{\partial P}{\partial V} \sigma_{V}\right)^{2}+\left(\frac{\partial P}{\partial I} \sigma_{I}\right)^{2}}$.

For a power level of $2 \mathrm{~W}$, the uncertainty in the power turns out to be

$\sigma_{p}= \pm \sqrt{(0.33 \times 0.1)^{2}+(6 \times 0.01)^{2}}$,

$\sigma_{p}= \pm 0.0684 \mathrm{~W}$.

The total power is given by

Table 2 Results of the uncertainty analysis

\begin{tabular}{llll}
\hline SI. no & Quantity measured & Uncertainty & Unit \\
\hline 1 & Temperature & \pm 0.2 & ${ }^{\circ} \mathrm{C}$ \\
2 & Voltage & \pm 0.1 & $\mathrm{~V}$ \\
3 & Current & \pm 0.01 & $\mathrm{~A}$ \\
4 & Power & \pm 2 & $\%$ \\
\hline
\end{tabular}


$P=P 1+P 2+P 3+P 4$,

$\sigma_{p}=2 \%$.

\section{Heat Transfer from the Heated Surface}

For the purpose of study, dimensionless numbers that characterize the heat transfer are defined below

Nusselt number $(\mathrm{Nu})$

In the present study, the Nusselt number reflects the time dependent relationship between heat flux and $\Delta T$ (Difference in base temperature and melting point). Thus, in the present study the heat flux remains constant with time, whereas $\Delta T$ keeps changing with time. The properties of $n$ eicosane are the same as those used in (Srikanth et al. 2015)

$\mathrm{Nu}=\frac{\mathrm{qH}}{\Delta T k}$.

Fourier number (Fo) Fourier number depicts the time for diffusion of heat across the PCM thickness and is defined as

$F_{o}=\frac{\alpha t}{H^{2}}$.

Stefan number (Ste) Stefan number represents the degree of superheating experienced by the liquid PCM The product of Ste and Fo represents the dimensionless time for phase change without convection.

Ste $=\frac{C_{p} q_{b} H}{L k}$.

Rayleigh number $(R a)$ To account for the buoyancy effect inside the liquid PCM that drives the heat transfer, Rayleigh number is included in the study. The Rayleigh number is also defined (Akhilesh et al. 2005) based on the PCM thickness.

$\mathrm{Ra}=\frac{g \beta \rho^{2} c_{\rho} q_{\rho} H^{4}}{k^{2} \mu}$.

The density and the dynamic viscosity of the PCM are taken at $309.7 \mathrm{~K}$ which corresponds to the melting temperature of the PCM.

\section{Heat Transfer Correlations}

In the present study, for a total wattage of 4 and $6 \mathrm{~W}$, a total of 110 experiments are performed and the time to reach set point was monitored. Randomly chosen four experimental results of temperature history are shown in Fig. 3. Table 3 shows the time to reach set point for randomly chosen 21 experiments. Using the experimental data, a correlation was obtained for the dimensionless time and dimensionless heat inputs.
Table 3 Time to reach set point temperature for 21 randomly chosen cases investigated experimentally

\begin{tabular}{lllllll}
\hline $\operatorname{Exp~ID~}$ & $\mathrm{Q}_{1}(\mathrm{~W})$ & $\mathrm{Q}_{2}(\mathrm{~W})$ & $\mathrm{Q}_{3}(\mathrm{~W})$ & $\mathrm{Q}_{4}(\mathrm{~W})$ & $\mathrm{t}_{c}(\mathrm{~s})$ & $\mathrm{t}_{d}(\mathrm{~s})$ \\
\hline 1 & 1.5 & 1.5 & 1.5 & 1.5 & 5830 & 13,145 \\
2 & 1.8 & 3 & 0.6 & 0.6 & 4880 & 12,395 \\
3 & 4.2 & 0.6 & 0.6 & 0.6 & 4705 & 15,305 \\
4 & 1.5 & 1.8 & 1.2 & 1.5 & 4550 & 11,430 \\
5 & 0.6 & 2.4 & 2.4 & 0.6 & 5215 & 16,035 \\
6 & 1 & 1 & 2 & 2 & 4660 & 20,875 \\
7 & 0.6 & 3.9 & 0.6 & 3.9 & 5255 & 17,860 \\
8 & 3 & 1 & 1 & 1 & 4200 & 11,945 \\
9 & 0.6 & 2.7 & 0.6 & 2.1 & 4970 & 16,905 \\
10 & 1 & 1 & 3 & 1 & 4680 & 11,700 \\
11 & 2.1 & 0.9 & 0.6 & 2.4 & 4415 & 20,475 \\
12 & 0.6 & 3.3 & 1.5 & 0.6 & 4865 & 25,000 \\
13 & 1.2 & 1.8 & 2.4 & 0.6 & 4505 & 23,315 \\
14 & 1.2 & 1.2 & 1.2 & 2.4 & 4610 & 26,000 \\
15 & 0.6 & 1.5 & 1.8 & 2.1 & 4455 & 25,050 \\
16 & 1.68 & 0.96 & 1.44 & 1.92 & 4350 & 28,000 \\
17 & 0.84 & 1.26 & 1.68 & 2.22 & 4415 & 19,850 \\
18 & 3.3 & 0.9 & 0.6 & 1.2 & 4475 & 28,500 \\
19 & 0.72 & 1.44 & 2.16 & 1.68 & 4620 & 21,500 \\
20 & 1.5 & 0.75 & 2.25 & 1.5 & 4130 & 27,500 \\
21 & 0.72 & 1.92 & 0.96 & 2.4 & 4260 & 29,000 \\
\hline & & & & & &
\end{tabular}

$q_{n}^{*}=\frac{q_{n}}{Q}$.

To include the effect of natural convection the Rayleigh number, $\mathrm{Ra}$ is included in the correlation.

$\frac{F o}{R a^{0.25}}=0.06 \times S t e^{-1} \times\left(q_{1}^{*}\right)^{0.024} \times\left(q_{2}^{*}\right)^{0.026} \times\left(q_{3}^{*}\right)^{-0.018}$.

The term on the left hand side of the correlation is the characteristic time for phase change with natural convection.

$\tau_{\text {fit }}=0.06 \times\left(q_{1}^{*}\right)^{0.024} \times\left(q_{2}^{*}\right)^{0.026} \times\left(q_{3}^{*}\right)^{-0.018}$.

Equation 10 is valid for the following range of parameters Valid for

$$
\begin{aligned}
& 1.27 \leq \text { Ste } \leq 1.92 \\
& 1.8 \times 10^{6} \leq R a \leq 2.8 \times 10^{6} \\
& 0.14 \leq q_{i}^{*} \leq \forall i=1,2 \text { and } 3
\end{aligned}
$$

In Eqs. 10 and 11, $\mathrm{q}_{4}$ is not included in the correlation since if $\mathrm{q}_{1} \mathrm{q}_{2} \mathrm{q}_{3}$ are specified $\mathrm{q}_{4}$ is automatically fixed to satisfy the constraint considered in the study.

Engineering usefulness of the correlation

Case 1

$q_{n}=4 W$. 
Case 2

$q_{n}=6 \mathrm{~W}$.

In order to avoid local overheating, let us assume that any individual heater can take a minimum of $10 \%$ of the total heat input and a maximum of $70 \%$ of the total heat input.

$0.1 \leq q_{n} * \leq 0.7$.

When the correlation is analyzed further

$\tau=0.06 \times q$.

$q=\left(q_{1}^{*}\right)^{0.024} \times\left(q_{2}^{*}\right)^{0.026} \times\left(q_{3}^{*}\right)^{-0.018}$,

$\left(q_{1} *\right)=\left(q_{2} *\right)=\left(q_{3} *\right)=0.25$.

In this case $q$ becomes 0.96 which is maximum and translates to the case of uniform heating with a melting time of $5830 \mathrm{~s}$.

For uniform heating case when any two heaters take $10 \%$ of the total heat input then the $\mathrm{q}$ is in the range of 0.91-0.93, which corresponds to a time of $5255 \mathrm{~s}$ which is close to the best performance The case with any two heaters receiving $10 \%$ of the total heat input and other two receiving 65 and $15 \%$ of the heat input is studied with the help of the correlation developed. Upon substitution in Eq. 11 we get $\tau$ as 0.95 . This value is very close to the uniform heating case. From this it is very clear that the negative exponent on one of the heat input ratio provides a damping effect on the characteristic time $\tau$. It can also stated that the value of $\tau$ does not increase with increase in power level of all the four heaters.

Additional experiments were conducted for the case of $5 \mathrm{~W}$ and these points were also added in the plot and are indicated as ' $*$ ' on the plot. It is seen that even for data not included in the development of the correlation the latter performs very well, buttressing the adequacy of the correlation and the results show good agreement as can be seen in the parity plot shown in Fig. 4.

We now consider the uniform heating case, which is equivalent to a single plate heater being used to heat the PCM. If the designer does not have the time or resources to carry out an exhaustive, intensive optimization process and assumes the uniform heating case for his or her design, it is important for him or her to know how much deviation in performance would occur with a variation about the uniform heating. Hence we consider a $10 \%$ variation above and below the uniform heating case of the four heaters and analyze the change in charging/discharging time. Considering a variation of $1.35-1.65 \mathrm{~W}$ in heating levels, $q$ becomes 0.95 which translates to the case melting time of $5530 \mathrm{~s}$. The charging time shows decrease of up to $5.8 \%$ (4 min). The discharging time shows a decrease of up to $4.3 \%$ (11 $\mathrm{min})$, both of which are not very significant.

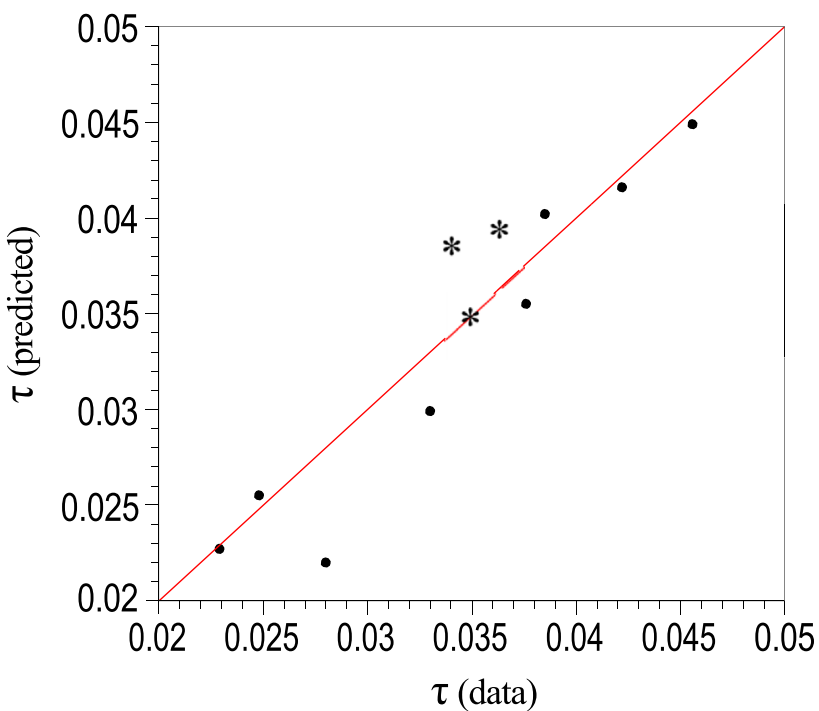

Fig. 4 Parity plot showing agreement between $\tau$ (data) and $\tau$ (predicted)

\section{Conclusions and Outlook}

Experiments were performed on a PCM based 72 pin fin heat sink with four discrete heaters at the base. The heat sink was made of aluminium and the PCM used was $n$ eicosane. Experiments for 110 different combination of heating level was conducted keeping the total power input equal to 4 and $6 \mathrm{~W}$. There was a significant diversity observed in the time to reach set point.

The salient conclusions/highlights of the study are

- The experiment is devoted to an interesting though practical application (electronic cooling) which is studied as an integral system.

- From the experimental findings, the correlations for the thermal performance (defined as the time to reach set point temperature) of this particular heat sink was developed as a function of the discrete power levels.

- Even for the case of non uniform heating, if the deviation of wattage of the individual heaters in $\pm 10 \%$ from the mean, the loss in the heat transfer performance during the charging and discharging is less than $5 \%$. In the view of the above, the correlation itself can be used to determine extreme cases of non uniform heating that need to be avoided, so that a significant loss of heat transfer performance can be avoided.

\section{References}

Akhilesh R, Narasimhan A, Balaji C (2005) Method to improve geometry for heat transfer enhancement in PCM composite heat sinks. Int J Heat Mass Transf 48:2759-2770 
Baby R, Balaji C (2012) Experimental investigations on phase change material based finned heat sinks for electronic equipment cooling. Int J Heat Mass Transf 55:1642-1649

Baby R, Balaji C (2013) A neural network-based optimization of thermal performance of phase change material-based finned heat sinks-an experimental study. Exp Heat Transf 26(5):431-452

Fan L-W, Xiao Y-Q, Zeng Y, Fang X, Wang X, Xu X, Yu Z-T, Hong $\mathrm{R}-\mathrm{H}$, Hu Y-C, Cen K-F (2013) Effects of melting temperature and the presence of internal fins on the performance of a phase change material (PCM)-based heat sink. Int J Therm Sci 70:114-126

Ho C-J, Viskanta R (1984) Heat transfer during melting from an isothermal vertical wall. ASME J Heat Transf 106:12-19

Jany P, Bejan A (1988) Scaling theory of melting with natural convection in an enclosure. Int $J$ Heat Mass Transf 31:1221-1235

Nayak K, Saha S, Srinivasan K, Dutta P (2006) A numerical model for heat sinks with phase change materials and thermal conductivity enhancers. Int J Heat Mass Transf 49:1833-1844
Pillai K, Brinkworth B (1976) The storage of low grade thermal energy using phase change materials. Appl Energy 2:205-216

Sasaguchi K, Viskanta R (1987) An experimental study of simultaneous melting and solidification around two horizontal, vertically spaced cylinders. Exp Heat Transf 1:223-236

Shokouhmand H, Kamkari B (2013) Experimental investigation on melting heat transfer characteristics of lauric acid in a rectangular thermal storage unit. Exp Therm Fluid Sci 50:201-212

Srikanth R, Balaji C (2017) Experimental investigation of thermal performance of phase change material based composite heat sinks with discrete heat sources. In: Proceedings of the 15th International Heat Transfer Conference, IHTC-15

Srikanth R, Nemani P, Balaji C (2015) Multi-objective geometric optimization of a PCM based matrix type composite heat sink. Appl Energy 156:703-714

Webb B, Viskanta R (1985) On the characteristic length scale for correlating melting heat transfer data. Int Commun Heat Mass Transf 12:637-646 